\title{
Severe Acute Systemic Reaction After the First Injections of Ixekizumab
}

\author{
John Pappas, MD; Maryam Liaqat, MD; Analisa V. Halpern, MD
}

\section{PRACTICE POINTS}

- Psoriasis is an autoimmune disorder with a predominance of $\mathrm{CD}^{+}$and $\mathrm{CD} 8^{+} \mathrm{T}$ cells that release cytokines, such as tumor necrosis factor $\alpha$ and interleukins, which promote inflammation in the skin and joints and is associated with systemic inflammation predisposing patients to cardiovascular disease.

- Common adverse effects of most biologic medications for psoriasis include injection-site pain and rash, fever, malaise, back pain, urticaria and flushing, edema, dyspnea, and nausea.

- Ixekizumab is a humanized IL-17A antagonist intended for adults with moderate to severe psoriasis. Certain rare side effects specific to ixekizumab include inflammatory bowel disease, thrombocytopenia, severe injection-site reactions, and candidiasis.

- Acute onycholysis and acute exacerbation of arthritis/ dactylitis are rare side effects of ixekizumab therapy.

We report the case of a 39-year-old woman who presented with generalized malaise; lymphadenopathy, arthritis; dactylitis; ecchymosis; acute onycholysis; and a red, nonpruritic, nonscaly, mottled rash on the right breast 24 hours after the first injections of ixekizumab for psoriasis and psoriatic arthritis. Ixekizumab is a humanized IgG4 monoclonal antibody that binds to IL-17A. Adverse events of ixekizumab include infection, inflammatory bowel disease, candidiasis and tinea infections, severe injection-site reactions, arthralgia, headache, infections, neutropenia, and thrombocytopenia. Other biologics, specifically tumor necrosis factor (TNF) inhibitors, have been reported to cause onycholysis attributed to immune dysregulation. We propose that ixekizumab alters the inflammatory cascade that underlies the induction of acute onycholysis and arthritis.

Cutis. 2022;109:E33-E35.

\section{Case Report}

A 39-year-old woman who was otherwise healthy presented with fatigue, malaise, a resolving rash, focal lymphadenopathy, increasing distal arthritis, dactylitis, resolving ecchymoses, and acute onycholysis of 1 week's duration that developed 13 days after initiating ixekizumab. The patient had a history of psoriasis and psoriatic arthritis for more than 10 years. She had been successfully treated in the past for psoriasis with adalimumab for several years; however, adalimumab was discontinued after an episode of Clostridium difficile colitis. The patient had a negative purified protein derivative (tuberculin) test prior to starting biologics as she works in the health care field. Routine follow-up purified protein derivative (tuberculin) test was positive. She discontinued all therapy for psoriasis and psoriatic arthritis prior to being appropriately treated for 6 months under the care of infectious disease physicians. She then had several pregnancies and chose to restart biologic treatment after weaning her third child from breastfeeding, as her skin and joint disease were notably flaring.

Ustekinumab was chosen to shift treatment away from tumor necrosis factor (TNF) $\alpha$ inhibitors. The patient's condition was under relatively good control for 1 year; however, she experienced notable gastrointestinal tract upset (ie, intermittent diarrhea and constipation), despite multiple negative tests for $C$ difficile. The patient was referred to see a gastroenterologist but never followed up. Due to long-term low-grade gastrointestinal problems, ustekinumab was discontinued, and the gastrointestinal symptoms resolved without treatment.

Given the side effects noted with TNF- $\alpha$ and IL-12/23 inhibitors and the fact that the patient's cutaneous and joint disease were notable, the decision was made to start

Dr. Pappas is from the Geisinger Commonwealth School of Medicine, Scranton, Pennsylvania. Dr. Liaqat is from Kaiser Permanente Medical Group, Santa Clara, California. Dr. Halpern is from the University of Pennsylvania, Philadelphia.

The authors report no conflict of interest.

Correspondence: Maryam Liaqat, MD, 710 Lawrence Expressway, Dept 472, Santa Clara, CA 95051 (Maryam.liaqat@kp.org). doi:10.12788/cutis. 0455 
the IL-17A inhibitor ixekizumab. The patient administered 2 injections, one in each thigh. Within 12 hours, she experienced severe injection-site pain. The pain was so severe that it woke her from sleep the night of the first injections. She then developed severe pain in the right axilla that limited upper extremity mobility. Within 48 hours, she developed an erythematous, nonpruritic, nonscaly, mottled rash on the right breast that began to resolve within 24 hours without treatment. In addition, 3 days after the injections, she developed ecchymoses on the trunk and extremities without any identifiable trauma, severe acute onycholysis in several fingernails (Figure 1) and toenails, dactylitis such that she could not wear her wedding ring, and a flare of psoriatic arthritis in the fingers and ankles.

At the current presentation (2 weeks after the injections), the patient reported malaise, flulike symptoms, and low-grade intermittent fevers. Results from a hematology panel displayed leukopenia at $2.69 \times 10^{3} / \mu \mathrm{L}$ (reference range, 3.54-9.06 $\left.\times 10^{3} / \mu \mathrm{L}\right)$ and thrombocytopenia at $114 \times 10^{3} / \mu \mathrm{L}$ (reference range, $165-415 \times 10^{3} / \mu \mathrm{L}$ ). ${ }^{1}$ Her most recent laboratory results before the ixekizumab injections displayed a white blood cell count level at $4.6 \times 10^{3} / \mu \mathrm{L}$ and platelet count at $159 \times 10^{3} / \mu \mathrm{L}$. C-reactive protein and erythrocyte sedimentation rate were within reference range. A shave biopsy of an erythematous nodule on the proximal interphalangeal joint of the fourth finger on the right hand displayed spongiotic dermatitis with eosinophils (Figure 2).

Interestingly, the psoriatic plaques on the scalp, trunk, and extremities had nearly completely resolved after only the first 2 injections. However, given the side effects, the second dose of ixekizumab was held, repeat laboratory tests were ordered to ensure normalization of cytopenia, and the patient was transitioned to pulse-dose topical steroids to control the remaining psoriatic plaques.

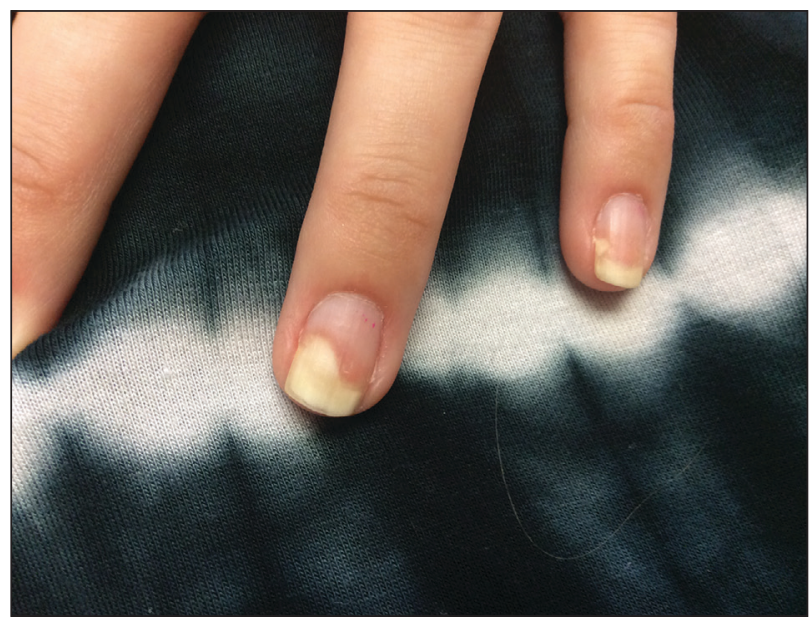

FIGURE 1. Severe acute onycholysis noted on the fourth and fifth fingernails of the left hand after ixekizumab injections for psoriasis and psoriatic arthritis.
One week after presentation ( 3 weeks after the initial injections), the patient's systemic symptoms had almost completely resolved, and she denied any further concerns. Her fingernails and toenails, however, continued to show the changes of onycholysis noted at the visit.

\section{Comment}

Ixekizumab is a human IgG4 monoclonal antibody that binds to IL-17A, one of the cytokines involved in the pathogenesis of psoriasis. The monoclonal antibody prevents its attachment to the IL-17 receptor, which inhibits the release of further cytokines and chemokines, decreasing the inflammatory and immune response. ${ }^{2}$

Ixekizumab was approved by the US Food and Drug Administration for plaque psoriasis after 3 clinical trialsUNCOVER-1, UNCOVER-2, and UNCOVER-3-were performed. In UNCOVER-3, the most common side effects that occurred-nasopharyngitis, upper respiratory tract infection, injection-site reaction, arthralgia, headache, and infections (specifically candidiasis) - generally were well tolerated. More serious adverse events included cardiovascular and cerebrovascular events, inflammatory bowel disease, and nonmelanoma skin cancer. ${ }^{3}$

Notable laboratory abnormalities that have been documented from ixekizumab include elevated liver function tests (eg, alanine aminotransferase, aspartate aminotransferase, bilirubin, and alkaline phosphatase), as well as leukopenia, neutropenia, and thrombocytopenia. ${ }^{4}$ Although short-term thrombocytopenia, as described in our patient, provides an explanation for the bruising noted on observation, it is unusual to note such notable ecchymoses within days of the first injection.

Onycholysis has not been documented as a side effect of ixekizumab; however, it has been reported as an adverse event from other biologic medications. Sfikakis et $\mathrm{al}^{5}$ reported 5 patients who developed psoriatic

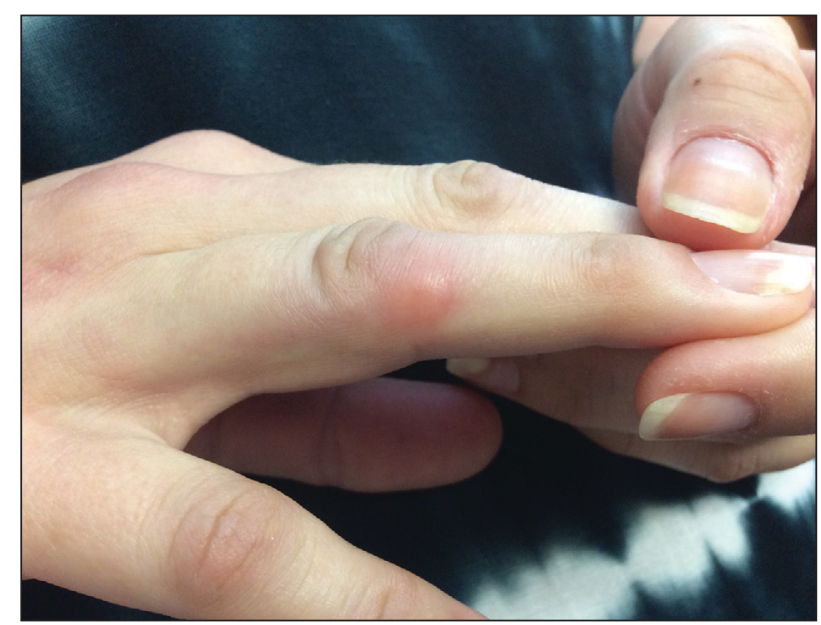

FIGURE 2. An erythematous nodule located on the proximal interphalangeal joint of the fourth finger on the right hand after ixekizumab injections for psoriasis and psoriatic arthritis. 
skin lesions after treatment with 3 different anti-TNF biologics-infliximab, adalimumab, or etanercept-for rheumatoid arthritis; 2 of those patients also developed nail changes consistent with psoriatic onycholysis. In all 5 patients, symptoms of rheumatoid arthritis improved despite the new-onset skin and nail psoriasis. ${ }^{5}$

The exact pathophysiology of these adverse events has not been clearly understood, but it has been proposed that anti-TNF biologics may initiate an autoimmune reaction in the skin and nails, leading to paradoxical psoriasis and nail changes such as onycholysis. Tumor necrosis factor may have a regulatory role in the skin that prevents autoreactive $\mathrm{T}$ cells, such as cutaneous lymphocyte antigen-expressing $\mathrm{T}$ cells that promote the formation of psoriasiform lesions. By inhibiting TNF, there can be an underlying activation of autoreactive $\mathrm{T}$ cells that leads to tissue destruction in the skin and nails. ${ }^{6}$ Anti-TNF biologics also could increase CXCR3, a chemokine receptor that allows autoreactive $\mathrm{T}$ cells to enter the skin and cause pathology.7

IL-17A and IL-17F also have been shown to upregulate the expression of TNF receptor II in synoviocytes, ${ }^{8}$ which demonstrates that IL-17 works in synergy with TNF- $\alpha$ to promote an inflammatory reaction. ${ }^{9}$ Due to the inhibitory effects of ixekizumab, psoriatic arthritis should theoretically improve. However, if there is an alteration in the inflammatory sequence, then the regulatory role of TNF could be suppressed and psoriatic arthritis could become exacerbated. Additionally, its associated symptoms, such as dactylitis, could develop, as seen in our patient. ${ }^{4}$ Because psoriatic arthritis is closely associated with nail changes of psoriasis, it is conceivable that acute arthritic flares and acute onycholysis are both induced by the same cytokine dysregulation. Further studies and a larger patient population need to be evaluated to determine the exact cause of the acute exacerbation of psoriatic arthritis with concomitant nail changes as noted in our patient.

Acute onycholysis (within 72 hours) is a rare side effect of ixekizumab. It can be postulated that our patient's severe acute onycholysis associated with a flare of psoriatic arthritis could be due to idiosyncratic immune dysregulation, promoting the activity of autoreactive $\mathrm{T}$ cells. The pharmacologic effects of ixekizumab occur through the inhibition of IL-17. We propose that by inhibiting IL-17 with associated TNF alterations, an altered inflammatory cascade could promote an autoimmune reaction leading to the described pathology.

\section{REFERENCES}

1. Kratz A, Pesce MA, Basner RC, et al. Laboratory values of clinical importance. In: Kasper D, Fauci A, Hauser S, et al, eds. Harrison's Principles of Internal Medicine. 19th ed. McGraw-Hill; 2014.

2. Ixekizumab. Package insert. Eli Lilly \& Co; 2017.

3. Gordon KB, Blauvelt A, Papp KA, et al. Phase 3 trials of ixekizumab in moderate-to-severe plaque psoriasis. N Engl J Med. 2016;375:345-356.

4. Leonardi C, Matheson R, Zachariae C, et al. Anti-interleukin-17 monoclonal antibody ixekizumab in chronic plaque psoriasis. $N$ Engl $\mathrm{J} \mathrm{Med.}$ 2012;366:1190-1199.

5. Sfikakis PP, Iliopoulos A, Elezoglou A, et al. Psoriasis induced by antitumor necrosis factor therapy: a paradoxical adverse reaction. Arthritis Rheum. 2005;52:2513-2518.

. Berg EL, Yoshino T, Rott LS, et al. The cutaneous lymphocyte antigen is a skin lymphocyte homing receptor for the vascular lectin endothelial cell-leukocyte adhesion molecule 1. J Exp Med. 1991;174:1461-1466.

7. Flier J, Boorsma DM, van Beek PJ, et al. Differential expression of CXCR3 targeting chemokines CXCL10, CXCL9, and CXCL11 in different types of skin inflammation. J Pathol. 2001;194:398-405.

8. Zrioual S, Ecochard R, Tournadre A, et al. Genome-wide comparison between IL-17A- and IL-17F-induced effects in human rheumatoid arthritis synoviocytes. J Immunol. 2009;182:3112-3120.

9. Gaffen SL. The role of interleukin-17 in the pathogenesis of rheumatoid arthritis. Curr Rheumatol Rep. 2009;11:365-370. 